\title{
Coupled Anti Multigroups: Some Properties
}

\author{
Clement Boateng Ampadu
}

31 Carrolton Road, Boston, MA 02132-6303, USA

e-mail: drampadu@hotmail.com

\begin{abstract}
Motivated by [1], the authors in [2] extended the notion of anti fuzzy groups to the multigroup context and studied some of their properties. In this paper we extend the work in a new direction termed coupled multigroup and obtain some new properties in this context. A conjecture concludes the paper.
\end{abstract}

\section{Some New Notions and Notations}

Definition 1.1. Let $X$ be a set. A coupled multiset $A$ over $X \times X$ will be a pair $<X \times X, C_{A}>$, where $C_{A}: X \times X \mapsto \mathbb{N} \cup\{0\}$ is a function such that for $(x, y) \in X \times X$ implies $A(x, y)$ is cardinal, and $A(x, y)=C_{A}(x, y)>0$, where $C_{A}(x, y)$ denotes the number of times an object $(x, y)$ occur in $A$, whenever $C_{A}(x, y)=0$ implies $(x, y) \notin X \times X$.

Let $B$ be a subset of $X \times X$ and define $1_{B}: X \times X \mapsto\{0,1\}$ by

$$
1_{B}(x, y)= \begin{cases}1 & (x, y) \in B \\ 0 & (x, y) \notin B .\end{cases}
$$

It follows that $B$ is a coupled multiset $\left\langle B, 1_{B}\right\rangle$, where $1_{B}$ is its characteristic function.

Remark 1.2. We call $X \times X$ the ground or generic set of the class of all coupled multisets containing objects from $X \times X$.

Received: June 24, 2020; Revised: July 5, 2020; Accepted: July 25, 2020

2010 Mathematics Subject Classification: 03E72, 06D72, 11E57, 19A22.

Keywords and phrases: multiset, multigroup, anti multigroup, coupled anti multigroup. 
Definition 1.3. Let $X \times X$ be the set from which coupled multisets are constructed. By $(X \times X)^{n}$ we mean the set of all coupled multisets of $X \times X$ such that no element occurs more than $n$ times.

Definition 1.4. Let $X \times X$ be the set from which coupled multisets are constructed. By $(X \times X)^{\infty}$ we mean the set of all coupled multisets of $X \times X$ such that there is no limit on the number of occurences of an element.

Notation 1.5. $C M S(X \times X)$ will denote the set of all coupled multisets over $X \times X$.

Remark 1.6. In this paper we focus on $C M S(X \times X)$ contained in $(X \times X)^{n}$.

Example 1.7. Let $X=\{a, b, c\}$ and

$$
X \times X=\{(a, a),(a, b),(a, c),(b, a),(b, b),(b, c),(c, a),(c, b),(c, c)\} .
$$

Then

$$
A=\left\{(a, a)^{2},(a, b)^{2},(a, c)^{2},(b, a)^{2},(b, b)^{2},(b, c)^{2},(c, a)^{3},(c, b)^{3},(c, c)^{3}\right\},
$$

where $(x, y)^{n}$ means $(x, y)$ repeated $n$ times, is a coupled multiset of $X \times X$.

Motivated by [3], we introduce the following

Definition 1.8. Let $X$ be a nonempty set, and $(X \times X)^{n}$ be the coupled multiset space defined over $X \times X$. For any

$$
A \in C M S(X \times X) \subseteq(X \times X)^{n}
$$

we define the complement of $A$ in $(X \times X)^{n}$, denoted $A^{c}$, by

$$
C_{A^{c}}(x, y)=n-C_{A}(x, y)
$$

for every $(x, y) \in X \times X$.

Remark 1.9. From now on $C M S(X \times X)$ will mean the set of all coupled multisets over $X \times X$ drawn from the coupled multiset space $(X \times X)^{n}$. 
Motivated by [4, we introduce the following

Definition 1.10. Let $A, B \in C M S(X \times X)$. We say $A$ is a coupled submultiset of $B$ written $A \subseteq B$, if

$$
C_{A}(x, y) \leq C_{B}(x, y)
$$

for all $(x, y) \in X \times X$. Also if $A \subseteq B$ and $A \neq B$, then we say $A$ is a proper coupled submultiset of $B$ written $A \subset B$. Moreover, we say a coupled multiset is the parent in relation to its coupled submultiset.

Motivated by [5], we introduce the following

Definition 1.11. Let $A, B \in C M S(X \times X), \wedge$ and $\vee$ denote minimum and maximum, respectively, and let $(x, y)$ be any object in $X \times X$.

(a) The intersection of $A$ and $B, A \cap B$, will be defined as

$$
C_{A \cap B}(x, y)=C_{A}(x, y) \wedge C_{B}(x, y) .
$$

(b) The union of $A$ and $B, A \cup B$, will be defined as

$$
C_{A \cup B}(x, y)=C_{A}(x, y) \vee C_{B}(x, y) .
$$

(c) The sum of $A$ and $B, A+B$, will be defined as

$$
C_{A+B}(x, y)=C_{A}(x, y)+C_{B}(x, y) .
$$

Motivated by [5], we introduce the following

Definition 1.12. Let $A, B \in C M S(X \times X)$. We say $A$ and $B$ are comparable to each other if and only if $B \subseteq A$ or $A \subseteq B$. Moreover, we say $A=B$ if and only if $C_{A}(x, y)=C_{B}(x, y)$ for all $(x, y) \in X \times X$.

Motivated by [6], we introduce the following 
Definition 1.13. Let $X$ be a group. A coupled multiset $A$ over $X \times X$ will be called a coupled multigroupoid of $X \times X$ if for all $(x, m),(y, v) \in X \times X$ we have

$$
C_{A}(x y, m v) \geq C_{A}(x, m) \wedge C_{A}(y, v)
$$

where $C_{A}$ denotes count function of $A$ from $X \times X$ into $\mathbb{N}$.

Motivated by [6] and [7] we introduce the following

Definition 1.14. Let $X$ be a group. A coupled multiset $A$ of $X \times X$ will be called a coupled multigroup of $X \times X$ if it satisfies the following two conditions

(a) $A$ is a coupled multigroupoid of $X \times X$.

(b) $C_{A}\left(x^{-1}, m^{-1}\right)=C_{A}(x, m)$ for all $(x, m) \in X \times X$.

Moreover, $C M G(X \times X)$ denotes the set of all coupled multigroups of $X \times X$

Motivated by [7], we have the following alternate characterization of coupled multigroups

Definition 1.15. Let $X$ be a group, and $A$ be a multiset over $X \times X$. If

$$
C_{A}\left(x y^{-1}, m v^{-1}\right) \geq C_{A}(x, m) \wedge C_{A}(y, m)
$$

for all $(x, m),(y, v) \in X \times X$, then $A$ is a coupled multigroup of $X \times X$.

Motivated by [6], we introduce the following

Definition 1.16. Let $A \in C M G(X \times X)$. A coupled submultiset $B$ of $A$ will be called a coupled submultigroup of $A$ denoted by $B \sqsubseteq A$ if $B$ is a coupled multigroup. A coupled submultigroup $B$ of $A$ will be called a proper coupled submultigroup denoted by $B \sqsubset A$, if $B \sqsubseteq A$ and $A \neq B$.

Motivated by [8], we introduce the following 
Definition 1.17. Let $A \in C M G(X \times X)$. By the strong upper cut of $A$, we mean the set

$$
A_{[n]}=\left\{(x, m) \in X \times X \mid C_{A}(x, m) \geq n, n \in \mathbb{N}\right\} .
$$

By the weak upper cut of $A$, we mean the set

$$
A_{(n)}=\left\{(x, m) \in X \times X \mid C_{A}(x, m) \geq n, n \in \mathbb{N}\right\} .
$$

Motivated by [7], we introduce the following

Definition 1.18. The inverse of an element $(x, m) \in X \times X$ in a coupled multigroup $A$ of $X \times X$ will be given by

$$
C_{A}\left(x^{-1}, m^{-1}\right)=C_{A^{-1}}(x, m)
$$

for all $(x, m) \in X \times X$.

In Section 3 of [2], the author presents anti-multigroup as a multigroup in reverse order. From now on we refine some of these concepts to the coupled anti multigroup setting and obtain some related properties in the next section.

Definition 1.19. Let $X$ be a groupoid. The coupled multiset $A$ of $X \times X$ will be called a coupled anti multigroupoid of $X \times X$ if

$$
C_{A}(x y, m v) \leq C_{A}(x, m) \vee C_{A}(y, v)
$$

for all $(x, m),(y, v) \in X \times X$.

Definition 1.20. A coupled multiset $A$ of $X \times X$ will be called a coupled anti multigroup of $X \times X$ if the following conditions hold:

(a) $C_{A}(x y, m v) \leq C_{A}(x, m) \vee C_{A}(y, v)$ for all $(x, m),(y, v) \in X \times X$.

(b) $C_{A}\left(x^{-1}, m^{-1}\right) \leq C_{A}(x, m)$ for all $(x, m) \in X \times X$.

Notation 1.21. The set of all coupled anti multigroups of $X \times X$ will be denoted by $C A M G(X \times X)$. 
Conjecture 1.22. Let $X=\{e, a, b, c\}$ be a group. Let the elements of $X \times X$ be such that

$$
\begin{aligned}
& (a, e)=(a, a)=(a, b)=(a, c) \\
& (b, e)=(b, a)=(b, b)=(b, c) \\
& (c, e)=(c, a)=(c, b)=(c, c) \\
& (e, e)=(e, a)=(e, b)=(e, c) .
\end{aligned}
$$

Assume we have the following

$$
\begin{aligned}
\left(a^{2}, e^{2}\right)=\left(b^{2}, e^{2}\right) & =\left(c^{2}, e^{2}\right)=(e, e) \\
\left(a b, e^{2}\right) & =(c, e) \\
\left(a c, e^{2}\right) & =(b, e) \\
\left(b c, e^{2}\right) & =(a, e) .
\end{aligned}
$$

Then the coupled multiset $A=\left\{\left(e^{2}, e^{2}\right),\left(a^{5}, e^{5}\right),\left(b^{4}, e^{4}\right),\left(c^{5}, e^{5}\right)\right\}$ is a coupled anti multigroup of $X \times X$.

In what follows we introduce a concept of cuts for coupled anti multigroups.

Definition 1.23. Let $A \in C A M G(X \times X)$. Then the set $\mathbb{A}_{[n]}$ for $n \in \mathbb{N}$ defined by

$$
\mathbb{A}_{[n]}=\left\{(x, m) \in X \times X \mid C_{A}(x, m) \leq n\right\}
$$

will be called the cut of $A$.

\section{Some Properties}

Proposition 2.1. Let $X$ be a nonempty set. If $A$ is a coupled multigroup of $X \times X$, then the following holds:

(a) $C_{A}\left(x^{-1}, m^{-1}\right)=C_{A}(x, m)$ for all $(x, m) \in X \times X$. 
(b) $C_{A}\left(e, e^{\prime}\right) \leq C_{A}(x, m)$ for all $(x, m) \in X \times X$, where $\left(e, e^{\prime}\right)$ is the identity element of $X \times X$.

(c) $C_{A}\left(x^{n}, m^{n}\right) \leq C_{A}(x, m)$ for all $(x, m) \in X \times X$ and $n \in \mathbb{N}$.

Proof. For (a): By Definition 1.20,

$$
C_{A}\left(x^{-1}, m^{-1}\right) \leq C_{A}(x, m)
$$

for all $(x, m) \in X \times X$. Also we have

$$
C_{A}(x, m) \leq C_{A}\left(\left(x^{-1}\right)^{-1},\left(m^{-1}\right)^{-1}\right) \leq C_{A}\left(x^{-1}, m^{-1}\right) .
$$

Combining the above two inequalities completes the proof.

For (b): Let $(x, m) \in X \times X$. Note that $x x^{-1}=e$ and $m m^{-1}=e^{\prime}$. It now follows that

$$
\begin{aligned}
C_{A}\left(e, e^{\prime}\right) & =C_{A}\left(x x^{-1}, m m^{-1}\right) \\
& \leq C_{A}(x, m) \vee C_{A}(x, m) \\
& =C_{A}(x, m)
\end{aligned}
$$

and (b) follows.

For (c): We have the following for all $n \in \mathbb{N}$,

$$
\begin{aligned}
C_{A}\left(x^{n}, m^{n}\right) & \leq C_{A}\left(x^{n-1}, m^{n-1}\right) \vee C_{A}(x, m) \\
& \leq C_{A}\left(x^{n-2}, m^{n-2}\right) \vee C_{A}(x, m) \vee C_{A}(x, m) \\
& \vdots \\
& \leq C_{A}(x, m) \vee \cdots \vee C_{A}(x, m) \\
& =C_{A}(x, m) .
\end{aligned}
$$


Proposition 2.2. If $A$ and $B$ are coupled anti multigroups of $X \times X$, then $A \cap B$ is a coupled anti multigroup of $X \times X$.

Proof. Let $(x, m),(y, v) \in X \times X$. Observe we have the following

$$
\begin{aligned}
C_{A \cap B}\left(x y^{-1}, m v^{-1}\right) & =C_{A}\left(x y^{-1}, m v^{-1}\right) \wedge C_{B}\left(x y^{-1}, m v^{-1}\right) \\
& \leq\left[C_{A}(x, m) \vee C_{A}(y, v)\right] \wedge\left[C_{B}(x, m) \vee C_{B}(y, v)\right] \\
& =\left[C_{A}(x, m) \wedge C_{B}(x, m)\right] \vee\left[C_{A}(y, v) \wedge C_{B}(y, v)\right] \\
& =C_{A \cap B}(x, m) \vee C_{A \cap B}(y, v) .
\end{aligned}
$$

Hence the conclusion.

Proposition 2.3. If $A$ and $B$ are coupled anti multigroups of $X \times X$, then the sum of $A$ and $B$ is a coupled multigroup of $X \times X$.

Proof. Let $(x, m),(y, v) \in X \times X$. Observe we have the following

$$
\begin{aligned}
C_{A+B}\left(x y^{-1}, m v^{-1}\right) & =C_{A}\left(x y^{-1}, m v^{-1}\right)+C_{B}\left(x y^{-1}, m v^{-1}\right) \\
& \leq\left[C_{A}(x, m) \vee C_{A}(y, v)\right]+\left[C_{B}(x, m) \vee C_{B}(y, v)\right] \\
& =\left[C_{A}(x, m)+C_{B}(x, m)\right] \vee\left[C_{A}(y, v)+C_{B}(y, v)\right] \\
& =C_{A+B}(x, m) \vee C_{A+B}(y, v) .
\end{aligned}
$$

Hence the conclusion.

Proposition 2.4. A coupled multiset $A$ is a coupled multigroup of $X \times X$ iff

$$
C_{A}\left(x y^{-1}, m v^{-1}\right) \leq C_{A}(x, m) \vee C_{A}(y, v)
$$

for all $(x, m),(y, v) \in X \times X$.

Proof. Assume $A$ is a coupled anti multigroup of $X \times X$. Then we know

$$
C_{A}(x y, m v) \leq C_{A}(x, m) \vee C_{A}(y, v)
$$

for all $(x, m),(y, v) \in X \times X$ and

$$
C_{A}\left(x^{-1}, m^{-1}\right) \leq C_{A}(x, m)
$$


for all $(x, m) \in X \times X$. By these conditions we have

$$
C_{A}\left(x y^{-1}, m v^{-1}\right) \leq C_{A}(x, m) \vee C_{A}(y, v)
$$

for all $(x, m),(y, v) \in X \times X$. Conversely, suppose the given condition is satisfied. By the following facts

$$
\begin{gathered}
C_{A}\left(e, e^{\prime}\right) \leq C_{A}(x, m) \\
C_{A}\left(x^{-1}, m^{-1}\right)=C_{A}(x, m)
\end{gathered}
$$

for all $(x, m) \in X \times X$, and

$$
\begin{aligned}
C_{A}(x y, m v) & \leq C_{A}\left[x\left(y^{-1}\right)^{-1}, m\left(v^{-1}\right)^{-1}\right] \\
& \leq C_{A}(x, m) \vee C_{A}\left(y^{-1}, v^{-1}\right) \\
& =C_{A}(x, m) \vee C_{A}(y, v)
\end{aligned}
$$

for all $(x, m),(y, v) \in X \times X$. It follows that $A$ is a coupled anti multigroup of $X \times X$.

Theorem 2.5. Let $X$ be a finite group, and $A$ be a coupled anti multigroupoid of $X \times X$. Then $A$ is a coupled anti multigroup.

Proof. Let $(x, m) \in X \times X,(x, m) \neq\left(e, e^{\prime}\right)$. Since $X$ is finite, $x$ and $m$ have finite order. Thus

$$
\left(x^{n}, m^{n}\right)=\left(e, e^{\prime}\right) \Longrightarrow\left(x^{-1}, m^{-1}\right)=\left(x^{n-1}, m^{n-1}\right) .
$$

By repeated application of the definition of coupled anti multigroupoid, we deduce the following

$$
\begin{aligned}
C_{A}\left(x^{-1}, m^{-1}\right) & =C_{A}\left(x^{n-1}, m^{n-1}\right) \\
& =C_{A}\left(x^{n-2} x, m^{n-2} m\right) \\
& \leq C_{A}\left(x^{n-2}, m^{n-2}\right) \vee C_{A}(x, m) \\
& \vdots \\
& \leq C_{A}(x, m) \vee \cdots \vee C_{A}(x, m) \\
& =C_{A}(x, m) .
\end{aligned}
$$

Hence the conclusion. 
Theorem 2.6. Let $A$ be a multiset of $X$. Then $A \in C M G(X \times X)$ iff $A^{c} \in$ $C A M G(X \times X)$.

Proof. Suppose $A \in C M G(X \times X)$. Then for all $(x, m),(y, v) \in X \times X$, we have

$$
C_{A}\left(x y^{-1}, m v^{-1}\right) \geq C_{A}(x, m) \wedge C_{A}(y, v)
$$

$\Longrightarrow$

$$
C_{\left(A^{c}\right)^{c}}\left(x y^{-1}, m v^{-1}\right) \geq C_{\left(A^{c}\right)^{c}}(x, m) \wedge C_{\left(A^{c}\right)^{c}}(y, v)
$$

$\Longrightarrow$

$$
1-C_{A^{c}}\left(x y^{-1}, m v^{-1}\right) \geq\left(1-C_{A^{c}}(x, m)\right) \wedge\left(1-C_{A^{c}}(y, v)\right)
$$

$\Longrightarrow$

$$
-C_{A^{c}}\left(x y^{-1}, m v^{-1}\right) \geq-1+\left[\left(1-C_{A^{c}}(x, m)\right) \wedge\left(1-C_{A^{c}}(y, v)\right)\right]
$$

$\Longrightarrow$

$$
C_{A^{c}}\left(x y^{-1}, m v^{-1}\right) \leq 1-\left[\left(1-C_{A^{c}}(x, m)\right) \wedge\left(1-C_{A^{c}}(y, v)\right)\right]
$$

$\Longrightarrow$

$$
\left.\left.C_{A^{c}}\left(x y^{-1}, m v^{-1}\right) \leq C_{A^{c}}(x, m)\right) \vee C_{A^{c}}(y, v)\right)
$$

$\Longrightarrow$

$$
A^{c} \in C A M G(X \times X) .
$$

Conversely suppose $A^{c}$ is a coupled anti multigroup of $X \times X$, then we have

$$
C_{A^{c}}\left(x y^{-1}, m v^{-1}\right) \leq C_{A^{c}}(x, m) \vee C_{A^{C}}(y, v)
$$

$\Longrightarrow$

$$
1-C_{A}\left(x y^{-1}, m v^{-1}\right) \leq\left(1-C_{A}(x, m) \vee\left(1-C_{A}(y, v)\right)\right.
$$

$\Longrightarrow$

$$
-C_{A}\left(x y^{-1}, m v^{-1}\right) \leq-1+\left[\left(1-C_{A}(x, m) \vee\left(1-C_{A}(y, v)\right)\right]\right.
$$

$\Longrightarrow$

$$
C_{A}\left(x y^{-1}, m v^{-1}\right) \geq 1-\left[\left(1-C_{A}(x, m) \vee\left(1-C_{A}(y, v)\right)\right]\right.
$$


$\Longrightarrow$

$$
C_{A}\left(x y^{-1}, m v^{-1}\right) \geq C_{A}(x, m) \wedge C_{A}(y, v)
$$

$\Longrightarrow$

$$
A \in C M G(X \times X)
$$

Proposition 2.7. Let $A \in C A M G(X \times X)$. If $C_{A}(x, m)>C_{A}(y, v)$ for some

$$
(x, m),(y, v) \in X \times X
$$

then

$$
C_{A}(x y, m v)=C_{A}(x, m)=C_{A}(y x, v m) .
$$

Proof. Suppose $C_{A}(x, m)>C_{A}(y, v)$ for some $(x, m),(y, v) \in X \times X$. Observe

$$
C_{A}(x y, m v) \leq C_{A}(x, m) \vee C_{A}(y, v)=C_{A}(x, m)
$$

Similarly

$$
\begin{aligned}
C_{A}(x, m) & =C_{A}\left(x y y^{-1}, m v v^{-1}\right) \\
& \leq C_{A}(x y, m v) \vee C_{A}(y, v) \\
& =C_{A}(x y, m v) .
\end{aligned}
$$

Thus, $C_{A}(x y, m v)=C_{A}(x, m)$. In a similar way, we have $C_{A}(y x, v m)=C_{A}(x, m)$. Hence, the conclusion.

Proposition 2.8. Let $A \in C A M G(X \times X)$. Then

$$
C_{A}\left(x y^{-1}, m v^{-1}\right)=C_{A}\left(e, e^{\prime}\right)
$$

iff $C_{A}(x, m)=C_{A}(y, v)$.

Proof. Assume $C_{A}\left(x y^{-1}, m v^{-1}\right)=C_{A}\left(e, e^{\prime}\right)$ for all $(x, m),(y, v) \in X \times X$, where $\left(e, e^{\prime}\right)$ is the identity of $X \times X$. Now observe we have the following 


$$
\begin{aligned}
C_{A}(x, m) & =C_{A}\left(x\left(y^{-1} y\right), m\left(v^{-1} v\right)\right) \\
& =C_{A}\left(\left(x y^{-1}\right) y,\left(m v^{-1}\right) v\right) \\
& \leq C_{A}\left(x y^{-1}, m v^{-1}\right) \vee C_{A}(y, v) \\
& =C_{A}(y, v) .
\end{aligned}
$$

Also we have

$$
\begin{aligned}
C_{A}(y, v) & =C_{A}\left[\left(x^{-1} x\right) y^{-1},\left(m^{-1} m\right) v^{-1}\right] \\
& =C_{A}\left[x^{-1}\left(x y^{-1}\right), m^{-1}\left(m v^{-1}\right)\right] \\
& \leq C_{A}(x, m) \vee C_{A}\left(x y^{-1}, m v^{-1}\right) \\
& \leq C_{A}(x, m) .
\end{aligned}
$$

Thus, $C_{A}(x, m)=C_{A}(y, m)$. For the converse, assume $C_{A}(x, m)=C_{A}(y, v)$ for all $(x, m),(y, v) \in X \times X$. Then we have

$$
C_{A}\left(x y^{-1}, m v^{-1}\right)=C_{A}\left(y y^{-1}, m m^{-1}\right)
$$

$\Longrightarrow$

$$
C_{A}\left(x y^{-1}, m v^{-1}\right)=C_{A}\left(e, e^{\prime}\right)
$$

Proposition 2.9. Let $A \in C A M G(X \times X)$. Then

$$
C_{A}(x y, m v) \leq C_{A}(y, v)
$$

for all $(x, m),(y, v) \in X \times X$ iff $C_{A}(x, m)=C_{A}\left(e, e^{\prime}\right)$.

Proof. Suppose $C_{A}(x y, m v)=C_{A}(y, v)$ for all $(x, m),(y, v) \in X \times X$. By letting $y=e$ and $v=e^{\prime}$, we obtain that

$$
C_{A}(x, m)=C_{A}\left(e, e^{\prime}\right)
$$


for all $(x, m) \in X \times X$. For the converse, suppose that $C_{A}(x, m)=C_{A}\left(e, e^{\prime}\right)$. Then $C_{A}(y, v) \geq C_{A}(x, m)$ and so

$$
C_{A}(x y, m v) \leq C_{A}(x, m) \vee C_{A}(y, v)=C_{A}(y, v) .
$$

Also

$$
\begin{aligned}
C_{A}(y, v) & =C_{A}\left(x^{-1} x y, m^{-1} m v\right) \\
& \leq C_{A}(x, m) \vee C_{A}(x y, m v) \\
& =C_{A}(x y, m v) .
\end{aligned}
$$

It follows that $C_{A}(x y, m v)=C_{A}(y, v)$ for all $(x, m),(y, v) \in X \times X$, finishing the proof.

Theorem 2.10. Let $A \in C A M G(X \times X)$. If $(x, m),(y, v) \in X \times X$ with $C_{A}(x, m) \neq$ $C_{A}(y, v)$, then

$$
C_{A}(x y, m v)=C_{A}(y x, v m)=C_{A}(x, m) \vee C_{A}(y, v) .
$$

Proof. Let $(x, m),(y, v) \in X \times X$. Since $C_{A}(x, m) \neq C_{A}(y, v), C_{A}(x, m)<C_{A}(y, v)$ or $C_{A}(y, v)<C_{A}(x, m)$. Suppose $C_{A}(x, m)<C_{A}(y, v)$, then $C_{A}(x y, m v) \leq C_{A}(y, v)$ and

$$
\begin{aligned}
C_{A}(y, v) & =C_{A}\left(x^{-1} x y, m^{-1} m v\right) \\
& \leq C_{A}\left(x^{-1}, m^{-1}\right) \vee C_{A}(x y, m v) \\
& =C_{A}(x, m) \vee C_{A}(x y, m v) \\
& =C_{A}(x y, m v) .
\end{aligned}
$$

Thus

$$
\begin{aligned}
C_{A}(y, v) & \leq C_{A}(x y, m v) \\
& \leq C_{A}(x, m) \vee C_{A}(y, v) \\
& =C_{A}(y, v) .
\end{aligned}
$$


From here, we have

$$
C_{A}(x y, m v) \leq C_{A}(x, m) \vee C_{A}(y, v)
$$

and

$$
C_{A}(x, m) \vee C_{A}(y, v) \leq C_{A}(x y, m v)
$$

which implies

$$
C_{A}(x y, m v)=C_{A}(x, m) \vee C_{A}(y, v) .
$$

Similarly, suppose $C_{A}(y, v)<C_{A}(x, m)$, then $C_{A}(y x, v m) \leq C_{A}(x, m)$ and

$$
\begin{aligned}
C_{A}(x, m) & =C_{A}\left(y^{-1} y x, v^{-1} v m\right) \\
& \leq C_{A}\left(y^{-1}, v^{-1}\right) \vee C_{A}(y x, v m) \\
& =C_{A}(y, v) \vee C_{A}(y x, v m) \\
& =C_{A}(y x, v m) .
\end{aligned}
$$

It now follows that

$$
\begin{aligned}
C_{A}(x, m) & \leq C_{A}(y x, v m) \\
& \leq C_{A}(y, v) \vee C_{A}(x, m) \\
& =C_{A}(x, m) .
\end{aligned}
$$

Therefore we have

$$
C_{A}(y x, v m)=C A(y, v) \vee C_{A}(x, m) .
$$

Hence the conclusion.

Corollary 2.11. If $A$ is a coupled anti multigroup of $X \times X$, then

$$
C_{A}(x y, m v)=C_{A}(x, m) \vee C_{A}(y, v)
$$

for all $(x, m),(y, v) \in X \times X$ with

$$
C_{A}(x, m) \neq C_{A}(y, v) .
$$


Proof. Let $(x, m),(y, v) \in X \times X$. Assume that $C_{A}(x, m)<C_{A}(y, m)$. Then

$$
C_{A}(x y, m v) \leq C_{A}(x, m) \vee C_{A}(y, v)=C_{A}(y, v)
$$

for all $(x, m),(y, v) \in X \times X$. Similarly,

$$
\begin{aligned}
C_{A}(x, m) \vee C_{A}(y, v) & =C_{A}\left(x^{-1} x y, m^{-1} m v\right) \\
& \leq C_{A}\left(x^{-1}, m^{-1}\right) \vee C_{A}(x y, m v) \\
& =C_{A}(x, m) \vee C_{A}(x y, m v) \\
& =C_{A}(x y, m v) .
\end{aligned}
$$

Hence

$$
C_{A}(x y, m v)=C_{A}(x, m) \vee C_{A}(y, v) .
$$

Proposition 2.12. Let $A$ be a coupled multigroup of $X \times X$. Then for any $n \in \mathbb{N}$ such that $n \geq C_{A}\left(e, e^{\prime}\right), \mathbb{A}_{[n]}$ is a subgroup of $X \times X$.

Proof. For all $(x, m),(y, v) \in \mathbb{A}_{[n]}$, we have

$$
C_{A}\left(x y^{-1}, m v^{-1}\right) \leq\left[C_{A}(x, m) \vee C_{A}(y, v)\right] \leq n .
$$

Hence the result.

Proposition 2.13. Let $A$ be a coupled multiset of $X \times X$ such that $\mathbb{A}_{[n]}$ is a subgroup of $X \times X$ for all $n \in \mathbb{N}$ with

$$
n \geq C_{A}\left(e, e^{\prime}\right) .
$$

Then $A$ is a coupled anti multigroup of $X \times X$.

Proof. Let $(x, m),(y, v) \in X \times X$ and $C_{A}(x, m)=n_{1}, C_{A}(y, v)=n_{2}$. Suppose $n_{2} \geq n_{1}$. Then $(x, m),(y, v) \in \mathbb{A}_{[n]}$, so that $\left(x y^{-1}, m v^{-1}\right) \in \mathbb{A}_{[n]}$. It follows that

$$
C_{A}\left(x y^{-1}, m v^{-1}\right) \leq n_{2}=n_{1} \vee n_{2}=C_{A}(x, m) \vee C_{A}(y, v) .
$$

Hence the result. 


\section{Open Problem}

We conjecture Theorem 3.14 of [9] can be proved in the setting of this paper. First we introduce the following

Definition 3.1. Let $A, B \in C M G(X \times X)$. If there exists $(y, v),\left(z, z^{\prime}\right) \in X \times X$ such that $x=y z$ and $m=v z^{\prime}$, then the product $A \circ B$ of $A$ and $B$ will be defined by

$$
C_{A \circ B}(x, m)=\bigvee_{x=y z, m=v z^{\prime}}\left(C_{A}(y, v) \wedge C_{B}\left(z, z^{\prime}\right)\right)
$$

otherwise

$$
C_{A \circ B}(x, m)=0 .
$$

In the setting of this paper, we claim Theorem 3.14 of [9] can be interpreted as follows

Conjecture 3.2. Let $X$ be a group. Suppose $A$ and $B$ are coupled multigroups of $X \times X$. Then

(a) $A \subseteq A \circ B$, if $C_{A}\left(e, e^{\prime}\right) \leq C_{B}\left(e, e^{\prime}\right)$.

(b) $A \subseteq A \circ B$ and $B \subseteq A \circ B$, if $C_{A}\left(e, e^{\prime}\right)=C_{B}\left(e, e^{\prime}\right)$.

\section{References}

[1] R. Biswas, Fuzzy subgroups and anti fuzzy subgroups, Fuzzy Sets Syst. 35 (1990), 121-124. https://doi.org/10.1016/0165-0114(90)90025-2

[2] P. A. Ejegwa, Concept of anti multigroups and its properties, Earthline J. Math. Sci. 4(1) (2020), 83-97. https://doi.org/10.34198/ejms.4120.8397

[3] S. P. Jena, S. K. Ghosh and B. K. Tripathy, On the theory of bags and lists, Inform. Sci. 132 (2001), 241-254. https://doi.org/10.1016/S0020-0255(01)00066-4

[4] A. Syropoulos, Mathematics of Multisets, Springer-Verlag Berlin Heidelberg, 2001, pp. 347-358. https://doi.org/10.1007/3-540-45523-X_17 
[5] D. Singh, A. M. Ibrahim, T. Yohanna and J. N. Singh, An overview of the applications of multisets, Novi Sad J. Math. 37(2) (2007), 73-92.

[6] P. A. Ejegwa and A. M. Ibrahim, Some group's analogous results in multigroup setting, Ann. Fuzzy Math. Inform. 17(3) (2019), 231-245. https://doi.org/10.30948/afmi.2019.17.3.231

[7] Sk. Nazmul, P. Majumdar and S. K. Samanta, On multisets and multigroups, Ann. Fuzzy Math. Inform. 6(3) (2013), 643-656.

[8] P. A. Ejegwa, Upper and lower cuts of multigroups, Prajna Int. J. Math. Sci. Appl. 1(1) (2017), 19-26

[9] P. A. Ejegwa and A. M. Ibrahim, Some properties of multigroups, Palestine Journal of Mathematics 9(1) (2020), 31-47.

This is an open access article distributed under the terms of the Creative Commons Attribution License (http://creativecommons.org/licenses/by/4.0/), which permits unrestricted, use, distribution and reproduction in any medium, or format for any purpose, even commercially provided the work is properly cited. 